\title{
Nanobiology-convergence of disciplines inspires great applications
}

\author{
Alexandra Kroll
}

Received: 19 September 2011/Revised: 29 September 2011/Accepted: 29 September 2011/Published online: 21 October 2011 (C) Springer Basel AG 2011

"Nanobiology is in its infancy" was concluded by Nussinov and Alleman [1] who presented an overview of the newly emerging field of research. Since then, 5 years of research have resulted in sophisticated applications and highly promising perspectives some of which are going to be reviewed in this compilation.

Nanobiology Molecular and cellular biology have always been based on the manipulation and study of structures on the nanoscale, however, the focus was not explicitly on the size-dependent properties of the structures of interest. Biophysicists, on the other hand, have been working on the quantification of molecular and cellular interactions of forces (Blow 2008). The intense development of nanotechnology and the increasing knowledge of physical properties of nanometer-sized objects have resulted in a new perspective on biological structures. Nanotechnology has enabled us to (a) vastly improve the analysis of biological structures and (b) to construct and manipulate biological objects on the nanoscale. These are at the same time the two main themes in what is now referred to as nanobiology: analysis and creation. The better the understanding of biological systems is on the nanoscale due to improved analysis tools, the better we can exploit this understanding to develop new bio-inspired or bio-mimicking devices or structures, possibly also for new analysis techniques. Nanobiology can be regarded as a convergence of biology and nanotechnology and is thus at

\footnotetext{
A. Kroll

CeNTech GmbH, Münster, Germany

A. Kroll $(\bowtie)$

Environmental Toxicology, Eawag, ETH Domain, Dübendorf, Switzerland

e-mail: alexandra.kroll@eawag.ch
}

the interface of biology, physics, chemistry, materials sciences, and engineering. Although different aspects of this field of research overlap with other disciplines, it provides a necessary frame for the focus on the size-dependent properties of biological structures and their application.

As nanobiology is a broad field of research, the editor of this multi-author review decided to focus on three selected fields of nanobiology described below. One common focus in all contributions is on health care, which has evolved to be one of the most important sectors of research and application.

Analytical tools The exact and reliable quantification of molecular interactions is an essential field of research. For instance, micrometer-scale cells interact with and attach to surfaces or neighboring cells via nanometer-scale structures. To understand the processes and parameters of attachment/detachment and interaction are important in fundamental research as well as in medical applications. Saitakis and Gizeli review the recent development of acoustic sensors for probing and quantifying these processes. The authors present an illustration of the acoustic systems used in animal cells studies and current applications in studying cellular physiology completed by an outlook on the potential of the technique in nanobiology.

Biological nanomaterials The study of biological nanometer-scale objects has inspired the design and assembly of biomolecules with defined properties. One new exciting and versatile tool are polymer nanoparticles. Aiertza et al. summarize the currently available techniques for the synthesis of the latter. The review explains the main characterization techniques and physical properties of single-chain polymer NPs and describes current and potential applications in nanomedicine.

Applications The range of applications of nanobiology is simply vast. From the beginning, one of the main areas of 
application of nanobiology has been health care, which results in a close link to nanomedicine. One of the driving ideas in this field is the development of therapeutic strategies that are effectively targeted to specific structures and function at the molecular level. Another main focus is on cost-efficient, specific, and especially, fast, diagnostics. Nanobiochips, for example, are versatile tools for molecular diagnostics that can lower the detection limit of conventional methods by orders of magnitude. The lab-ona-chip will have a significant impact for the development of point-of-care diagnostics. Valiokas et al. review the available techniques for the fabrication of nanobiochips including suitable platforms, materials for functionalization, and functionalization procedures. The authors also provide an overview of the literature on diverse functional nanobiochips. Kurkina and Balasubramanian then go into detail on the sensing techniques used on nanobiochips and more specific, functionalization protocols. The review also provides an overview of nanobiochips successfully applied to realistic samples. In the last section of this MAR, Galvin et al. present case studies on engineered nanoparticles for drug delivery in cancer and cardiovascular disease treatment. Synthetic nanoparticles have been improved to efficiently encapsulate and release drugs and to deliver them precisely to target cells leading to reduced sideeffects and lower dosage.

The recent progress reviewed in the following contributions highlights the interdisciplinarity in the field of nanobiology that allows us to work more efficiently and precise in many applications.

Acknowledgments This multi-author review was compiled as a follow-up of the NanoBioEurope 2010 Conference. AK thanks the authors for their cooperation and excellent contributions.

\section{Reference}

1. Nussinov R, Alemán C (2006) Nanobiology: from physics and engineering to biology. Phys Biol 3(1) 